\title{
Control of Laser Induced Stress for Diffractive Optics in Transparent Media
}

\author{
Qi Sun, Timothy Lee, Martynas Beresna and Gilberto Brambilla \\ Optoelectronics Research Centre, University of Southampton, Southampton, SO17 1BJ, United Kingdom
}

The femtosecond laser writing technique has been widely exploited for transparent materials processing. One of the useful features following laser irradiation is refractive index modification. The sign of refractive index change depends on irradiation conditions. Negative refractive index change can be $10^{-2}$, but it is accompanied with strong optical scattering. The laser induced positive refractive index change is about $10^{-3}$. For diffractive optics this is relatively small change and thus requires to modify a large physical thickness for inducing a significant optical phase delay. This can be achieved with Bessel beams [1]. However, an extended Bessel zone leads to higher stress around the laser affected zone, which can induce structural cracks with a high energy deposition level [2]. The large stressed region also distorts subsequent writing pulses which detriments the overall uniformity of the inscribed structure limiting the performance of the written diffractive element. Here, we report a method for controlling stress accumulated during the inscription with a femtosecond Bessel beam inside silica, and demonstrate its benefits for improving structural uniformity and preventing crack formation.

As shown in Fig. 1(a), the $515 \mathrm{~nm}$ wavelength laser beam (200 kHz repetition rate, $200 \mathrm{fs}$ pulse duration) was generated as the second harmonic from a $\lambda=1030 \mathrm{~nm}$ beam with a BBO crystal. An axicon with a $179^{\circ}$ apex angle was then used to obtain the Bessel beam. During writing, a computer controlled translation stage moved the silica substrate precisely in the $x$ and $y$ directions, while the objective (0.4NA) was adjusted in the vertical $z$ direction. Structures were written with typical pulse density of $10^{5}$ pulses $/ \mathrm{mm}$ and pulse energy of $0.75 \mu \mathrm{J}$.

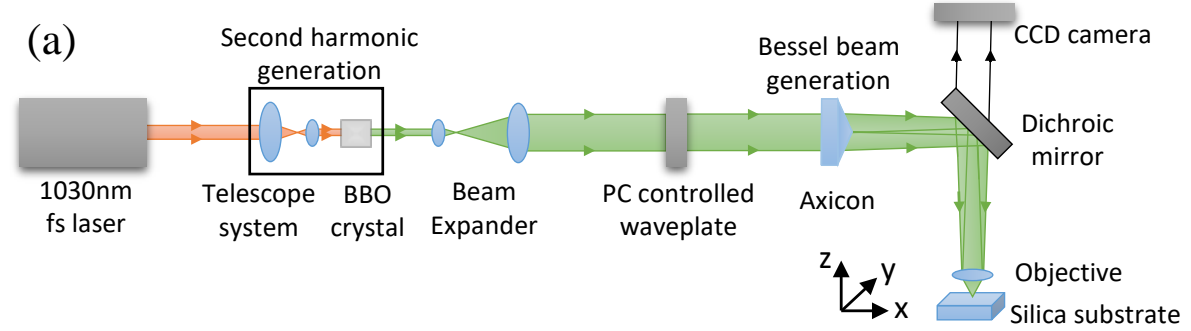

(b)

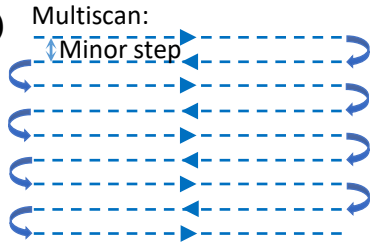

(c)

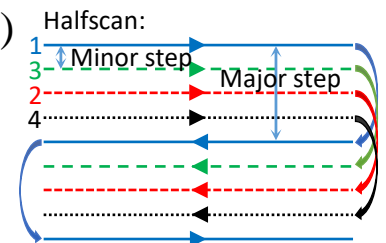

(d)

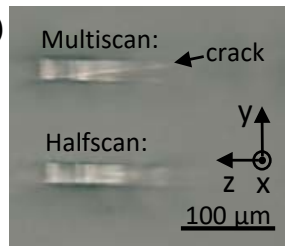

Fig. 1 (a) Fs laser writing experimental setup including Gaussian-Bessel beam generation. (b) Standard multiscan laser writing method. (c) Halfscan laser writing method, colors denote different writing order (blue-red-green-black). (d) Microscope image of cross section of fs laser written waveguide. Laser scan starts from top to bottom. Top waveguide written with multiscan method has a large crack due to stress accumulation; bottom waveguide is written with halfscan method which has a more even profile. Waveguide width (in y direction) is $20 \mu \mathrm{m}$.

During material processing with the commonly used multiscan writing method [3] (Fig. 1(b)), the material stress gradually builds up on one side due to closely spaced $(200 \mathrm{~nm})$ lines which results in a non-uniform refractive index increase and a large crack along the cross-section (see top waveguide in Fig. 1(d)). To reduce the asymmetry of stress, we use a new 'halfscan' method depicted in Fig. 1(c) where the line order is rearranged. Different colours stand for different writing order (blue-red-green-black). The main principle is to keep each scan jump at a major step $(0.8 \mu \mathrm{m})$ and finally achieve an overall minor step structure. As each subsequent scan line has a larger gap than the multiscan method, this reduces the effect of the previous scan line on the writing of the next line. Also, the larger scan step reduces the chances of damage. The bottom waveguide in Fig. 1(d) shows the improved profile with halfscan writing method. We then exploited the halfscan method to write 50/50 gratings; the first order diffraction efficiency improved by $39 \%$ (from $\eta=18 \%$ to $25 \%$ ) compared to multiscan method. For more complex multilayer structures, we also demonstrated a 2-layer Fresnel lens with focal efficiency of 55\% (with the theoretical limit of 65\%) with halfscan method.

\section{References}

[1] M. Mikutis, T. Kudrius, G. Slekys, D. Paipulas, and S. Juodkazis, "High 90\% effciency Bragg gratings formed in fused silica by femtosecond Gauss-Bessel laser beams," Opt. Materials Express 3, 1862-1871 (2013).

[2] Audrey Champion, Martynas Beresna, Peter Kazansky, and Yves Bellouard, "Stress distribution around femtosecond laser affected zones: effect of nanogratings orientation," Opt. Express 21, 24942-24951 (2013).

[3] Y. Nasu, M. Kohtoku, and Y. Hibino, "Low-loss waveguides written with a femtosecond laser for flexible interconnection in a planar light-wave circuit," Opt. Lett. 30, 723-725 (2005). 